\title{
EXPONENTIAL DECAY FOR SC-GRADIENT FLOW LINES
}

\author{
PETER ALBERS AND URS FRAUENFELDER
}

\begin{abstract}
In this paper we introduce the notion of sc-action functionals and their scgradient flow lines. Our approach is inspired by Floer's unregularized gradient flow. The main result of this paper is that under a Morse condition sc-gradient flow lines have uniform exponential decay towards critical points. The ultimate goal for the future is to construct a M-polyfold bundle over a M-polyfold such that the space of broken sc-gradient flow lines is the zero set of a appropriate sc-section. Here uniform exponential decay is essential.

Of independent interest is that we derive exponential decay estimates using interpolation inequalities as opposed to Sobolev inequalities. An advantage is that interpolation inequalities are independent of the dimension of the source space.
\end{abstract}

\section{Contents}

1. Introduction

2. Sc-action functionals and sc-gradient flow lines

3. Exponential decay for sc-gradient flow lines

4. An example

Appendix A. Interpolation inequalities

Appendix B. Sobolev vs. interpolation

References

\section{INTRODUCTION}

In Morse homology one considers a chain complex generated by the critical points of a Morse function and with a boundary operator defined by counting isolated gradient flow lines between critical points. In certain favorable circumstances this recipe even works on infinite dimensional Hilbert manifolds, e.g. counting closed geodesics on a Riemannian manifold where the Hilbert manifold in question is the loop space of the manifold. A generalization of this situation are Hamiltonian dynamical systems which admit a Lagrangian formulation, see for instance AM05] for a modern treatment. Sometimes Hilbert space methods work also in the Hamiltonian formulation, see for instance the celebrated proof by Conley-Zehnder [CZ83] of the Arnold conjecture on the torus. So far generalizing the beautiful arguments by ConleyZehnder to general symplectic manifolds failed. Instead, the breakthrough came with Floer's idea of using the unregularized gradient flow equation, see [Flo88.

Recently Hofer-Wysocki-Zehnder HWZ07, HWZ09a, HWZ09b discovered a new notion of smoothness in infinite dimensions called scale-smoothness which still features the chain rule and tangent functors. Thus, it gives rise to new manifold structures in infinite dimensions

This material is supported by the SFB 878 Groups, Geometry and Actions (PA) and by the Alexander von Humboldt Foundation (UF). 
such as sc-manifolds and more generally M-polyfolds. In this paper we introduce the notion of sc-action functionals and their sc-gradient flow lines. Our approach is inspired by Floer's unregularized gradient flow. The main result of this paper is that under a Morse condition sc-gradient flow lines have uniform exponential decay towards critical points. The ultimate goal for the future is to construct a M-polyfold bundle over a M-polyfold such that the space of broken sc-gradient flow lines is the zero set of a appropriate sc-section. Here uniform exponential decay is essential. The main result is Theorem 3.1 below.

Folk knowledge asserts that near Morse critical points gradient flow lines decay exponentially fast. One of the purposes of this article is to present a framework in which the previous statement is true. For this the sc-language by Hofer-Wysocki-Zehnder HWZ07, HWZ09a, HWZ09b is essential, indeed it enables us to define a good notion of a Morse critical point. Moreover, sc-spaces appeared (in different form) in interpolation theory, see e.g. [BL76, Tri78]. We make use of interpolation inequalities to prove exponential decay. In particular, the proof does not use special features of the underlying gradient flow equation. Thus, this approach generalizes the statement but sharply contrast the method of proof in previous works on exponential decay in Morse and Floer theory such as [Sch93, Sal99, RS01, Zil09]

\section{SC-ACTION FUnCTIONALS AND SC-GRADIENT FLOW LINES}

In this paper we work with sc-spaces as introduced by Hofer-Wysocki-Zehnder in [HWZ07. Let

$$
\mathbb{E}=\left(E_{k}\right)_{k \in \mathbb{N}}
$$

be a sc-space where each $E_{k}$ is a Hilbert space. We denote by $\langle\cdot, \cdot\rangle$ the Hilbert-space inner product on $E_{0}$ and by $\|\cdot\|_{k}$ the norm on $E_{k}$.

Remark 2.1. $\mathbb{E}$ is called a sc-Hilbert space, see [HWZ07, Theorem 3.10].

We abbreviate

$$
\mathbb{E}^{m}:=\left(E_{k+m}\right)_{k \in \mathbb{N}}
$$

Next we define the notion of a sc-action funtionals which is inspired by action functionals treated in Floer theory. For this let $U \subset E_{0}$ be an open set. $U$ induces a sc-Hilbert structure $\mathbb{U}:=\left(U_{k}\right)_{k \in \mathbb{N}}$ with

$$
U_{k}:=U \cap E_{k}
$$

In [HWZ07] Hofer-Wysocki-Zehnder introduce the notion of sc-smoothness on sc-spaces. part of the definition of sc-space is the compactness and density of the inclusion $E_{k+1} \hookrightarrow$ $E_{k}$ which implies the chain rule for sc-smooth maps, see [HWZ07, Theorem 2.16]. This is absolutely crucial for what follows.

Definition 2.2. A sc-smooth map

$$
A: \mathbb{U}^{1} \rightarrow \mathbb{R}
$$

is a sc-action functional if it admits a gradient. That is, a sc-smooth vector field $\nabla A: \mathbb{U}^{1} \rightarrow \mathbb{E}$ satisfying

In particular,

$$
\langle\nabla A(x), \xi\rangle=D A(x) \xi \quad \forall x \in U_{2}, \xi \in E_{1}
$$

$$
\nabla A\left(U_{k+1}\right) \subset E_{k}
$$

The set of critical points of $A$ is

$$
\operatorname{Crit} A:=\left\{x \in U_{\infty} \mid D A(x)=0\right\}=\left\{x \in U_{\infty} \mid \nabla A(x)=0\right\} \subset U_{\infty} .
$$


Remark 2.3. Because of the denseness of the embedding $U_{2} \hookrightarrow U_{1}$ the gradient, if it exists, is uniquely defined. On the other hand not every sc-smooth function admits a gradient, e.g. the map $x \mapsto\langle x, y\rangle$ where $y \in E_{0} \backslash E_{1}$ is fixed, is sc-smooth but does not admit a gradient. In fact, it is even $C^{\infty}$ in the classical sense when restricted to any level $E_{k}$.

By definition the critical points are smooth points. However, there might be non-smooth $x \in U \backslash U_{\infty}$ with $D A(x)=0$, see Section 4. In Floer theory the critical point equation is elliptic and therefore critical points are automatically smooth.

Definition 2.4. An sc-action functional $A$ is called Morse if the Hessian

$$
\mathscr{H}_{A}=D \nabla A: U_{k+2} \times E_{k+1} \rightarrow E_{k},
$$

is for $k \in \mathbb{N}$ an isomorphism at critical points $x \in \operatorname{Crit} A$, i.e.

$$
\nabla A(x)=0 \quad \Longrightarrow \quad \mathscr{H}_{A}(x): E_{k+1} \stackrel{\cong}{\longrightarrow} E_{k} \quad \forall k \geq 0 .
$$

Remark 2.5. The existence of a Morse critical point immediately implies that the sc-Hilbert space $\mathbb{E}$ is fractal, see Fra09a and Fra09b, i.e. sc-isomorphic to

$$
\left(\ell^{2} \supset \ell_{f}^{2} \subset \ell_{f^{2}}^{2} \supset \cdots\right)
$$

for some unbounded monotone increasing function $f: \mathbb{N} \rightarrow \mathbb{R}_{>0}$. Here $\ell_{f}^{2} \equiv \ell_{f}^{2}(\mathbb{N})$ is the space

$$
\left\{\left(a_{n}\right) \in \ell^{2}(\mathbb{N}) \mid \sum_{n=1}^{\infty} f(n) a_{n}^{2}<\infty\right\} .
$$

We point out that there exists a common orthogonal basis $\left(e_{\nu}\right)_{\nu} \in E_{\infty}$ for all $E_{n}$, e.g. the image of the standard basis of $\ell^{2}$.

A concrete example of a sc-action functional to keep in mind is

$$
E_{k}:=\ell_{f^{k}}^{2}(\mathbb{Z}):=\left\{\left(a_{n}\right) \in \ell^{2}(\mathbb{Z}) \mid \sum_{n=-\infty}^{\infty}(f(n))^{k} a_{n}^{2}<\infty\right\}
$$

with $f(n):=n^{2}+1$. Then $A\left(a_{n}\right):=\sum_{n=-\infty}^{\infty} n a_{n}^{2}$ is a sc-action functional. Indeed the gradient

$$
\nabla A\left(a_{n}\right)=\sum_{n=-\infty}^{\infty} 2 n a_{n} e_{n}
$$

is a sc-vector field. Moreover,

$$
\operatorname{Crit} A=\left\{\left(a_{n}\right) \mid a_{n}=0 \text { if } n \neq 0\right\} .
$$

If we identify $H^{1}\left(S^{1}, \mathbb{C}\right) \equiv \ell_{f}^{2}(\mathbb{Z})$ via Fourier series the sc-action functional $A$ corresponds to the symplectic area functional. It is important to note that the inner product is defined on $E_{0}$ whereas the sc-action functional make sense only on $E_{1}$.

Lemma 2.6. Let $A$ be a Morse sc-action functional. Then critical points of $A$ are isolated in $E_{10}$, in particular also in $E_{\infty}$.

Proof. We give here a nonstandard proof. This will be useful in the proof of the actionenergy inequality in Proposition 2.7. We first define the energy functional $e: \mathbb{U}^{1} \rightarrow \mathbb{R}$ associated with the sc-action functional $A$

$$
e(x):=\langle\nabla A(x), \nabla A(x)\rangle
$$


which is sc-smooth since the $A$ is sc-smooth and the map $(\xi, \eta) \mapsto\langle\xi, \eta\rangle$ is sc-smooth. We observe that

$$
e(x)=0 \quad \text { if and only if } D A(x)=0 .
$$

For simplicity we assume that the critical point is $0 \in \operatorname{Crit} A$, then

$$
D^{2} e(0)[\xi, \eta]=2\left\langle\mathscr{H}_{A}(0) \xi, \mathscr{H}_{A}(0) \eta\right\rangle .
$$

It follows from [HWZ07, Proposition 2.14] that

$$
e: U_{4}=\left(\mathbb{U}^{1}\right)_{3} \rightarrow \mathbb{R}
$$

is a $C^{3}$-function. Thus by Taylor's formula we can write

$$
\begin{aligned}
e(x) & =\frac{1}{2} D^{2} e(0)(x, x)+\mathcal{O}\left(\|x\|_{4}^{3}\right) \\
& =\left\|\mathscr{H}_{A}(0) x\right\|_{0}^{2}+\mathcal{O}\left(\|x\|_{4}^{3}\right) \\
& \geq C\left(\|x\|_{1}^{2}-\|x\|_{4}^{3}\right)
\end{aligned}
$$

where we used that $\mathscr{H}_{A}(0)$ is an isomorphism. In view of the interpolation inequality A.11) we continue

$$
e(x) \geq C\left(\|x\|_{1}^{2}-C^{\prime}\|x\|_{10}\|x\|_{1}^{2}\right)=C\left(1-C^{\prime}\|x\|_{10}\right)\|x\|_{1}^{2} .
$$

Thus, if $\|x\|_{10}$ is sufficiently small then $e(x)>0$ and thus $x \notin \operatorname{Crit} A$. In particular, $0 \in \operatorname{Crit} A$ is isolated. This proves the Lemma.

Proposition 2.7 (Action-Energy Inequality). We assume that $x^{+} \in \operatorname{Crit} A$ with $A\left(x^{+}\right)=a^{+}$. Then there exists $\epsilon=\epsilon\left(x^{+}\right)>0$ and $\kappa=\kappa\left(x^{+}\right)>0$ such that

$$
\left|A(x)-a^{+}\right| \leq \frac{e(x)}{\kappa} \quad \forall\left\|x-x^{+}\right\|_{10}<\epsilon .
$$

Remark 2.8. If $A$ is the symplectic area functional the action-energy inequality is a consequence of the isoperimetric inequality. We use the action-energy inequality to prove exponential decay for sc-gradient flow lines. This approach has been used before in GS05] and [Zil09].

Proof. Without loss of generality we assume that $x^{+}=0 \in \operatorname{Crit} A$ with $A(0)=0$. [HWZ07, Proposition 2.14] implies that $A: U_{4} \rightarrow \mathbb{R}$ is $C^{3}$ and thus

$$
|A(x)| \leq\left|\left\langle\mathscr{H}_{A}(0) x, x\right\rangle\right|+C_{1}\|x\|_{4}^{3}
$$

for sufficiently small $x$. Since $\mathscr{H}_{A}(0)$ is bounded we can estimate further using (A.11)

$$
|A(x)| \leq C_{2}\left(1+\|x\|_{10}\right)\|x\|_{1}^{2}
$$

and employing inequality (2.20)

$$
|A(x)| \leq \frac{C_{2}\left(1+\|x\|_{10}\right)}{C_{3}\left(1-C_{4}\|x\|_{10}\right)} e(x) .
$$

Thus, for $\|x\|_{10}$ sufficiently small the Proposition follows.

Definition 2.9. A (negative) sc-gradient flow line of $A$ is a sc-smooth map $x: \mathbb{R} \rightarrow \mathbb{U}$ satisfying

$$
x^{\prime}(s)=-\nabla A(x(s))
$$

where

$$
x^{\prime}(s):=D x(s) 1 .
$$


Remark 2.10. Since $\mathbb{R}$ carries only the constant scale structure the requirement of being smooth for a sc-gradient flow line $x: \mathbb{R} \rightarrow \mathbb{U}$ implies that $x(\mathbb{R}) \subset U_{\infty}$.

\section{EXPONENTIAL DECAY FOR SC-GRADIENT FLOW LINES}

In this section we assume that $A: \mathbb{U}^{1} \rightarrow \mathbb{R}$ is a Morse sc-action functional. Moreover, let $x: \mathbb{R} \rightarrow \mathbb{E}$ be a sc-gradient flow line such that

$$
\lim _{s \rightarrow+\infty} x(s)=x^{+} \in \operatorname{Crit} A
$$

where convergence is with respect to the $E_{\infty}$-topology. The main result of this paper is that $E_{\infty}$-convergence together with the Morse property imply uniform exponential convergence. In the following we abbreviate

$$
x^{(m)}:=\frac{d^{m} x}{d s^{m}} \quad \forall m \in \mathbb{N} .
$$

for a sc-gradient flow line $x: \mathbb{R} \rightarrow \mathbb{E}$.

Theorem 3.1 (Uniform exponential decay). Let $\kappa$ be the constant from Proposition 2.7 and $0<\kappa^{\prime}<\frac{\kappa}{3}$. Then there constants $C_{j, m}=C_{j, m}\left(\kappa^{\prime}\right)>0, j, m \in \mathbb{N}$, such that

$$
\left\|x(s)-x^{+}\right\|_{j} \leq C_{j, 0} e^{-\kappa^{\prime} s} \quad \forall s \geq 0
$$

and

$$
\left\|x^{(m)}(s)\right\|_{j} \leq C_{j, m} e^{-\kappa^{\prime} s} \quad \forall s \geq 0, m \geq 1 .
$$

As a first step we prove the following Proposition.

Proposition 3.2. Let $\kappa$ be the constant from Proposition 2.7 and $0<\kappa^{\prime}<\frac{\kappa}{2}$. Then there constants $C_{j}=C_{j}\left(\kappa^{\prime}\right)>0, j \in \mathbb{N}$, such that

$$
\left\|x(s)-x^{+}\right\|_{j} \leq C_{j} e^{-\kappa^{\prime} s} \quad \forall s \geq 0
$$

Furthermore, there exists a constant $C>0$

$$
A(x(s))-a^{+} \leq C e^{-\kappa s} \quad \forall s \geq 0
$$

where $a^{+}=A\left(x^{+}\right)$.

Proof. As a first step we claim that

$$
\left\|x(s)-x^{+}\right\|_{0} \leq C_{0} e^{-\frac{\kappa}{2} s} \quad \forall s \geq 0
$$

where $\kappa$ is the constant from Proposition 2.7. For this we fix $s_{0} \in \mathbb{R}$ such that

$$
\left\|x(s)-x^{+}\right\|_{10}<\epsilon^{+} \quad \forall s \geq s_{0},
$$

where $\epsilon^{+}$is assumed to be smaller than $\epsilon\left(x^{+}\right)$from Proposition 2.7 and such that there is no other critical point of $A$ which has $\|\cdot\|_{10}$-distance to $x^{+}$less than $\epsilon^{+}$. Next we prove that if there exists $s_{1} \in \mathbb{R}$ with $x\left(s_{1}\right)=x^{+}$then $x(s)=x^{+}$for all $s \geq s_{1}$ (and thus in this case the desired estimate follows trivially.) If the last assertion is wrong then we can find $s_{2}>s_{1}$ with 
$x\left(s_{2}\right) \neq x^{+}$. On the other hand the action $A$ strictly decreases along non-constant sc-gradient flow lines:

$$
\begin{aligned}
A\left(x\left(s_{2}\right)\right)-A\left(x\left(s_{1}\right)\right) & =\int_{s_{1}}^{s_{2}} \frac{d}{d s} A(x(s)) d s \\
& =\int_{s_{1}}^{s_{2}}\left\langle\nabla A(x(s)), x^{\prime}(s)\right\rangle d s \\
& =-\int_{s_{1}}^{s_{2}}\left\|x^{\prime}(s)\right\|_{0}^{2} d s \\
& <0 .
\end{aligned}
$$

In the same way we conclude

$$
A(x(s)) \leq A\left(x\left(s_{2}\right)\right) \quad \forall s \geq s_{2} .
$$

In particular, we arrive at the contradiction

$$
A\left(x^{+}\right) \leq A\left(x\left(s_{2}\right)\right)<A\left(x\left(s_{1}\right)\right)=A\left(x^{+}\right) .
$$

Therefore, from now on we assume without loss of generality that

$$
x(s) \neq x^{+} \quad \forall s \geq s_{0} .
$$

Since by assumption there are no other critical points in a $\epsilon^{+}$-neighborhood of $x^{+}$(measured with the $\|\cdot\|_{10}$-norm) and since $x(\mathbb{R}) \subset E_{\infty}$ we conclude that

$$
\nabla A(x(s)) \neq 0 \quad \forall s \geq s_{0} .
$$

Next we prove the following estimate in this situation:

$$
\left\|x^{+}-x(s)\right\|_{0} \leq \frac{2}{\sqrt{\kappa}} \sqrt{A(x(s))-a^{+}}
$$


for all $s \geq s_{0}$. Indeed:

$$
\begin{aligned}
& \left\|x^{+}-x(s)\right\|_{0} \leq \int_{s}^{\infty}\left\|x^{\prime}(t)\right\|_{0} d t \\
& =\int_{s}^{\infty}\|\nabla A(x(t))\|_{0} d t \\
& =\int_{s}^{\infty} \frac{\|\nabla A(x(t))\|_{0}^{2}}{\|\nabla A(x(t))\|_{0}} d t \\
& =\int_{s}^{\infty} \frac{\|\nabla A(x(t))\|_{0}^{2}}{\sqrt{e(x(t))}} d t \\
& \leq \frac{1}{\sqrt{\kappa}} \int_{s}^{\infty} \frac{\|\nabla A(x(t))\|_{0}^{2}}{\sqrt{A(x(t))-a^{+}}} d t \\
& =-\frac{1}{\sqrt{\kappa}} \int_{s}^{\infty} \frac{\left\langle\nabla A(x(t)), x^{\prime}(t)\right\rangle}{\sqrt{A(x(t))-a^{+}}} d t \\
& =-\frac{1}{\sqrt{\kappa}} \int_{s}^{\infty} \frac{D A(x(t)) x^{\prime}(t)}{\sqrt{A(x(t))-a^{+}}} d t \\
& =-\frac{1}{\sqrt{\kappa}} \int_{s}^{\infty} \frac{\frac{d}{d t} A(x(t))}{\sqrt{A(x(t))-a^{+}}} d t \\
& =-\frac{1}{\sqrt{\kappa}} \int_{s}^{\infty} \frac{\frac{d}{d t}\left(A(x(t))-a^{+}\right)}{\sqrt{A(x(t))-a^{+}}} d t \\
& =-\frac{2}{\sqrt{\kappa}} \int_{s}^{\infty} \frac{d}{d t} \sqrt{A(x(t))-a^{+}} d t \\
& =-\frac{2}{\sqrt{\kappa}}\left(\sqrt{A\left(x^{+}\right)-a^{+}}-\sqrt{A(x(s))-a^{+}}\right) \\
& =\frac{2}{\sqrt{\kappa}} \sqrt{A(x(s))-a^{+}}
\end{aligned}
$$

according to the definition of the energy, see (2.15), and Proposition 2.7. Next we show that $A(x(s))$ converges exponentially fast to $a^{+}$. For this observe

$$
\begin{aligned}
\frac{d}{d s} A(x(s)) & =D A(x(s)) x^{\prime}(s) \\
& =\left\langle\nabla A(x(s)), x^{\prime}(s)\right\rangle \\
& =-\langle\nabla A(x(s)), \nabla A(x(s))\rangle \\
& =-e(x(s))
\end{aligned}
$$

and thus, since $\left|A(x(s))-a^{+}\right|=A(x(s))-a^{+}$, we get from Proposition 2.7

$$
\begin{aligned}
\frac{d}{d s}\left(A(x(s))-a^{+}\right) & =-e(x(s)) \\
& \leq-\kappa\left(A(x(s))-a^{+}\right) .
\end{aligned}
$$

This implies that there exists $C>0$

$$
A(x(s))-a^{+} \leq C e^{-\kappa s} \quad \forall s \geq s_{0}
$$


as claimed in the statement. Combining these estimates we get

$$
\left\|x^{+}-x(s)\right\|_{0} \leq \frac{2}{\sqrt{\kappa}} \sqrt{A(x(s))-a^{+}} \leq C_{0} e^{-\frac{\kappa}{2} s} \quad \forall s \geq 0
$$

for some $C_{0}>0$. It remains to establish the Theorem for the $\|\cdot\|_{j}$-norms. A special case of the interpolation inequality (A.1) is

$$
\|x(s)\|_{j} \leq C_{j, L}\|x(s)\|_{L}^{\frac{j}{L}}\|x(s)\|_{0}^{\frac{L-j}{L}} .
$$

Given $0<\kappa^{\prime}<\frac{\kappa}{2}$ choosing $L$ sufficiently large and using that $\|x(s)\|_{L}$ converges to 0 (for any $L)$ we obtain

$$
\|x(s)\|_{j} \leq C_{j} e^{-\kappa^{\prime} s} \quad \forall s \geq 0
$$

This proves the Proposition.

To extend Proposition 3.2 to higher derivatives of the sc-gradient flow line we need the following Lemma.

Lemma 3.3. If there exists $\kappa>0, T>0$ and $C>0$ such that

$$
\int_{T}^{\infty}\left\|x^{\prime}(s)\right\|_{0}^{2} d s \leq C e^{-\kappa T}
$$

and moreover if $\left\|x^{\prime \prime}(s)\right\|_{0}<\epsilon$ for all $s \geq T$ then

$$
\left\|x^{\prime}(T)\right\|_{0}^{2} \leq \sqrt[3]{8 \epsilon C e^{-\kappa T}}
$$

Proof. We set

$$
\mu:=\left\|x^{\prime}(T)\right\|_{0}
$$

Then for $t \in\left[T, T+\frac{\mu}{2 \epsilon}\right]$ we estimate

$$
\begin{aligned}
\left\|x^{\prime}(t)\right\|_{0} & \geq\left\|x^{\prime}(T)\right\|_{0}-\int_{T}^{T+\frac{\mu}{2 \epsilon}}\left\|x^{\prime \prime}(s)\right\|_{0} d s \\
& \geq \frac{\mu}{2}
\end{aligned}
$$

and therefore

$$
\begin{aligned}
C e^{-\kappa T} & \geq \int_{T}^{\infty}\left\|x^{\prime}(s)\right\|_{0}^{2} d s \\
& \geq \int_{T}^{T+\frac{\mu}{2 \epsilon}}\left\|x^{\prime}(s)\right\|_{0}^{2} d s \\
& \geq \frac{\mu^{3}}{8 \epsilon} .
\end{aligned}
$$

Thus,

$$
\left\|x^{\prime}(T)\right\|_{0} \leq \sqrt[3]{8 \epsilon C e^{-\kappa T}}
$$

as claimed.

Remark 3.4. Let $T: \mathbb{U} \oplus \mathbb{E} \rightarrow \mathbb{E}$ be $s c^{0}$ and linear in the second entry. Then for every $x \in U_{k}$, there exists a neighborhood $V_{x} \subset U_{k}$ of $x$ and a constant $c_{k}>0$ such that

$$
\|T(y, \cdot)\| \leq c_{k} \quad \forall y \in V_{x}
$$


where $\|T(y, \cdot)\|$ denotes the $E_{k}-E_{k}$-operator norm. That is, the operator norm of $T(y, \cdot)$ is locally bounded. Indeed, since $T$ is $s c^{0}$ there exists $\delta>0$ and a neighborhood $V_{x} \subset U_{k}$ such that

$$
T\left(V_{x} \times B_{\delta}(0)\right) \subset B_{1}(0)
$$

where $B_{r}(0)$ is the $r$-ball in $E_{k}$. This implies that $\|T(y, \cdot)\|<\frac{1}{\delta}$ for all $y \in V_{x}$. In particular, if $K \subset U$ is compact, then the operator norm $\|T(x, \cdot)\|, x \in K$, is uniformly bounded.

Now we prove Theorem 3.1 .

Proof of Theorem 3.1. We already established the assertion for $x(s)$ for all $j$ in Proposition 3.2. We proceed by a bootstrapping argument. To start the bootstrapping we recall from Proposition 3.2 that there exists a constant $C>0$ with

$$
A(x(s))-a^{+} \leq C e^{-\kappa s} \quad \forall s \geq s_{0}
$$

where $a^{+}=A\left(x^{+}\right)$. We estimate

$$
\begin{aligned}
\int_{T}^{\infty}\left\|x^{\prime}(s)\right\|^{2} d s & =-\int_{T}^{\infty} D A(x(s)) x^{\prime}(s) d s \\
& =A(x(T))-a^{+} \\
& \leq C e^{-\kappa T}
\end{aligned}
$$

and thus by Lemma 3.3 we conclude

$$
\left\|x^{\prime}(T)\right\| \leq C^{\prime} e^{-\frac{\kappa}{3} T} .
$$

Deriving the equation $x^{\prime}=-\nabla A(x)$ gives

$$
x^{(k)}=\sum_{j=1}^{k-1} \sum_{\substack{l_{1} \geq l_{2} \geq \ldots \geq l_{j} \\ \sum_{r=1}^{j} l_{r}=k-1}} C\left(l_{1}, \ldots l_{j}\right) D^{j} \nabla A(x)\left(x^{\left(l_{1}\right)}, x^{\left(l_{2}\right)}, \ldots, x^{\left(l_{j}\right)}\right)
$$

with some combinatorial constants $C\left(l_{1}, \ldots l_{j}\right) \geq 1$. For $x \in E_{j+1}$ the operator norm of $D^{j} \nabla A(x): E_{j} \times \ldots \times E_{j} \rightarrow E_{0}$ is locally bounded, see Remark 3.4. Since $x(\mathbb{R}) \cup\left\{x^{ \pm}\right\}$is compact the operator norm of $D^{j} \nabla A(x(s))$ is uniformly bounded in $s \in \mathbb{R}$. Thus, by induction uniform exponential decay for $x^{\prime}$ implies uniform exponential decay for $x^{(k)}$ and therefore the Theorem.

\section{An exAmple}

An easy example to consider is the symplectic area functional perturbed by a smooth Hamiltonian term. The symplectic area functional is a quadratic functional, see Remark 2.5, and the perturbation is very tame namely an $s c^{+}$-operator. Thus, it is very easy to see that if critical points of the perturbed symplectic action functional are Morse in the usual sense they are sc-Morse and gradient flow lines decay exponentially fast, see for instance Sal99.

Therefore, in this section we study a sc-action functional which is not an $s c^{+}$-perturbation of a quadratic functional. For this we fix $f(n)=n^{2}: \mathbb{N}_{\geq 1} \rightarrow \mathbb{N}_{\geq 1}$ and consider the sc-space $\ell^{2} \supset \ell_{f}^{2} \supset \ell_{f^{2}}^{2} \cdots$ with

$$
\ell_{f}^{2}:=\left\{\left(a_{n}\right)_{n \geq 1} \mid a_{n} \in \mathbb{R}, \sum_{n=1}^{\infty} f(n) a_{n}^{2}<\infty\right\} .
$$


We set

$$
\begin{aligned}
A: \ell_{f}^{2} & \rightarrow \mathbb{R} \\
\left(a_{n}\right) & \mapsto \sum_{n=1}^{\infty}\left(n a_{n}^{2}+n^{3} a_{n}^{3}\right)
\end{aligned}
$$

and claim that $A$ is a sc-action functional. The map $\left(a_{n}\right) \mapsto \sum n a_{n}^{2}$ is a sc-action functional, see Remark 2.5. Since sc-action functionals form a vector space it remains to check that $\left(a_{n}\right) \mapsto B(a):=\sum_{n=1}^{\infty} n^{3} a_{n}^{3}$ is a sc-action functional where $a=\left(a_{n}\right)$.

In general it holds

$$
\left\|\left(a_{n}\right)\right\|_{\ell^{p}} \leq\left\|\left(a_{n}\right)\right\|_{\ell^{q}}
$$

for $p \geq q$. Thus, we conclude

$$
\sum_{n=1}^{\infty} n^{3} a_{n}^{3}=\left\|\left(n a_{n}\right)\right\|_{\ell^{3}}^{3} \leq\left\|\left(n a_{n}\right)\right\|_{\ell^{2}}^{3}=\left\|a_{n}\right\|_{\ell_{f}^{2}}^{3}<\infty,
$$

in particular, $B: \ell_{f}^{2} \rightarrow \mathbb{R}$ is well-defined. Moreover, using the same inequality it follows that

$$
\left.B\right|_{\ell^{2} k}: \ell_{f^{k}}^{2} \rightarrow \mathbb{R}
$$

is smooth in the classical sense, i.e. $B$ is $C^{\infty}$ on every level. Thus, it follows from [HWZ07, Proposition 2.15] that $B$ is sc-smooth. The candidate for the gradient of $B$ is

$$
\nabla B(a)=\sum_{n=1}^{\infty} 3 n^{3} a_{n}^{2} e_{n}
$$

where $\left\{e_{n}\right\}$ is the standard orthonormal basis of $\ell^{2}$. It remains to check that $\nabla B$ is sc-smooth. The first derivative is given by

$$
D \nabla B(a) \hat{a}=\sum_{n=1}^{\infty} 6 n^{3} a_{n} \hat{a}_{n} e_{n}
$$

which is a well-defined continuous map $D \nabla B: \ell_{f^{k+1}}^{2} \times \ell_{f^{k}}^{2} \rightarrow \ell_{f^{k-1}}^{2}$. Indeed, if $a \in \ell_{f^{k+1}}^{2}$ i.e. $\sum_{n=1}^{\infty} n^{2 k+2} a_{n}^{2}<\infty$ and $\hat{a} \in \ell_{f^{k}}^{2}$ i.e. $\sum_{n=1}^{\infty} n^{2 k} \hat{a}_{n}^{2}<\infty$ then

$$
\begin{aligned}
\sum_{n=1}^{\infty} n^{2 k-2} n^{6} a_{n}^{2} \hat{a}_{n}^{2} & \leq \sum_{n=1}^{\infty} n^{2 k+2} a_{n}^{2} n^{2 k} \hat{a}_{n}^{2} \\
& \leq\left(\sum_{n=1}^{\infty} n^{2 k+2} a_{n}^{2}\right)\left(\sum_{n=1}^{\infty} n^{2 k} \hat{a}_{n}^{2}\right) \\
& <\infty
\end{aligned}
$$

Similarly it follows that $D^{2} \nabla B$ is well-defined and continuous. The higher derivatives vanish. Thus, $B$ is sc-smooth.

The gradient of $A$ is given by

$$
\nabla A(a)=\sum_{n=1}^{\infty}\left(2 n a_{n} e_{n}+3 n^{3} a_{n}^{2} e_{n}\right) .
$$

The critical point equation is

$$
2 a_{n}+3 n^{2} a_{n}^{2}=0,
$$


thus

$$
a_{n}=0 \quad \text { or } \quad a_{n}=-\frac{2}{3 n^{2}} .
$$

Therefore, there are uncountably many solutions. However since we require that critical points are smooth the only critical points are those solutions where only finitely many $a_{n}$ are non-zero. In fact if infinitely many $a_{n}$ are non-zero then $\left(a_{n}\right) \notin \ell_{f^{2}}^{2}$. In particular, there are only countably many critical points.

Remark 4.1. We point out that $0 \in \operatorname{Crit} A$ is not an isolated critical point in the $\ell_{f}^{2}$ topology. In particular, there cannot be a Morse Lemma on $\ell_{f}^{2}$. Nevertheless, $A$ is a Morse sc-action functional since at $a=0$ we have

$$
\mathscr{H}_{A}(0)\left(\hat{a}_{n}\right)=\sum_{n=1}^{\infty} 2 n \hat{a}_{n} e_{n} .
$$

In general, if $a \in \operatorname{Crit} A$ with $a_{i}=-\frac{2}{3 i^{2}}$ for $i \in I \subset \mathbb{N}$ and $I$ finite then

$$
\mathscr{H}_{A}(a)\left(\hat{a}_{n}\right)=\sum_{n \notin I} 2 n \hat{a}_{n} e_{n}-\sum_{n \in I} 2 n \hat{a}_{n} e_{n}
$$

Thus, the Morse index of $a$ equals $|I|$ in particular all critical points have finite Morse index but infinite Morse coindex.

Similarly, one can replace the index set $\mathbb{N}$ by $\mathbb{Z}$. Then all critical points have infinite Morse index and coindex. But, as in Floer theory, the index differences are finite.

Since the gradient of $A$ is of diagonal form a gradient flow line $x=\left(x_{n}\right): \mathbb{R} \rightarrow \ell_{\infty}^{2}$ satisfies

$$
x_{n}^{\prime}(s)=-2 n x_{n}(s)-3 n^{3}\left(x_{n}(s)\right)^{2} .
$$

This is a special case of a Bernoulli ODE with solution

$$
x_{n}(s)=\frac{1}{c_{n} e^{2 n s}-\frac{3}{2} n^{2}}
$$

where $c_{n}$ is the integration constant. As to be expected from our discussion of the Morse indices the only finite energy solution solution converges at $+\infty$ to 0 and at $-\infty$ to $-\frac{2}{3 n^{2}}$. It does so exponentially fast uniformly on all levels and with all derivatives.

\section{Appendix A. Interpolation INEQUALities}

The following Proposition is a special case of the interpolation theorem by Stein-Weiss.

Proposition A.1. Let $\mathbb{E}$ be a fractal sc-space, see Remark 2.5. Then for $0 \leq i<j<k$

$$
\|x\|_{j} \leq C_{i, j, k}\|x\|_{k}^{\frac{j-i}{k-i}}\|x\|_{i}^{\frac{k-j}{k-i}} \quad \forall x \in E_{i} .
$$

Proof. By definition of being fractal we may assume without loss of generality that

$$
\mathbb{E}=\left(\ell^{2} \supset \ell_{f}^{2} \subset \ell_{f^{2}}^{2} \supset \cdots\right)
$$

for some unbounded monotone increasing function $f: \mathbb{N} \rightarrow \mathbb{R}_{>0}$. Here $\ell_{f}^{2}$ is the space

$$
\left\{\left(a_{n}\right) \in \ell^{2} \mid \sum_{n} f(n) a_{n}^{2}<\infty\right\} .
$$

Then the statement follows by combining Theorem 1.18.5 and Theorem 1.3.3(g) in [Tri78. In the notation of the book choose $A=\mathbb{R}, X=\mathbb{N}$, and $\mu$ the counting measure, i.e. every 
point has measure one. Then we can apply Theorem 1.18 .5 with $p=p_{0}=p_{1}=2, w_{0}^{2}:=f^{k}$, $w_{1}^{2}:=f^{i}$, and $\theta:=\frac{k-j}{k-i}$. After that Theorem 1.3.3(g) relates the norm on the space $A_{0}=$ $L_{2, w_{0}^{2}(x)}(\mathbb{R})=\ell_{f^{k}}^{2}$ and $A_{1}=\ell_{f^{i}}^{2}$.

We now present an elementary proof of Proposition A.1 which relies on the fractal property of the sc-space $\mathbb{E}$. Namely we note that there exists a natural sc-isomorphism (even a scisometry)

$$
\mathbb{E} \cong \mathbb{E}^{m}
$$

for all $m \geq 0$. The proof shows that the constants $C_{i, j, k}=1$.

An elementary proof of Proposition A.1. We proceed in three steps.

Step 1: $\|x\|_{1} \leq\|x\|_{2}^{1 / 2}\|x\|_{0}^{1 / 2}$ for every $x \in \ell_{f^{2}}^{2}$.

We equivalently show

$$
\|x\|_{1}^{4} \leq\|x\|_{2}^{2}\|x\|_{0}^{2}, \quad \forall x \in \ell_{f^{2}}^{2} .
$$

To see that we estimate

$$
\begin{aligned}
\|x\|_{1}^{4} & =\left(\sum_{i=1}^{\infty} f(i) x_{i}^{2}\right)^{2} \\
& =\sum_{i=1}^{\infty} f(i)^{2} x_{i}^{4}+2 \sum_{i<j} f(i) f(j) x_{i}^{2} x_{j}^{2} \\
& \leq \sum_{i=1}^{\infty} f(i)^{2} x_{i}^{4}+\sum_{i<j}\left(f(i)^{2}+f(j)^{2}\right) x_{i}^{2} x_{j}^{2} \\
& =\left(\sum_{i=1}^{\infty} f(i)^{2} x_{i}^{2}\right)\left(\sum_{j=1}^{\infty} x_{j}^{2}\right) \\
& =\|x\|_{2}^{2}\|x\|_{0}^{2} .
\end{aligned}
$$

This finishes the proof of Step 1.

Step 2: We prove the theorem if $i=0$.

The proof is by a induction on $k$. For $k \geq 2$ denote by $(A)_{k}$ the assertion for every $j \in\{1, \ldots, k-1\}$. The assertion $(A)_{2}$ is already true in view of Step 1. Hence it suffices to explain the induction step. For that purpose suppose that $(A)_{k-1}$ is true. By induction hypothesis we have

as well as

$$
\|x\|_{1} \leq\|x\|_{k-1}^{\frac{1}{k-1}}\|x\|_{0}^{\frac{k-2}{k-1}}
$$

$$
\|x\|_{k-2} \leq\|x\|_{k-1}^{\frac{k-2}{k-1}}\|x\|_{0}^{\frac{1}{k-1}} .
$$

Now observe that $\ell_{f}^{2}$ is isometric to $\ell^{2}$ via the map $\left\{x_{\nu}\right\} \mapsto\left\{f(\nu)^{1 / 2} x_{\nu}\right\}$. This maps restricts for every $m \in \mathbb{N}$ to an isometry $\ell_{f^{m+1}}^{2} \rightarrow \ell_{f^{m}}^{2}$. Therefore we obtain from (A.6) by shifting the inequality

$$
\|x\|_{k-1} \leq\|x\|_{k}^{\frac{k-2}{k-1}}\|x\|_{1}^{\frac{1}{k-1}}
$$


Using (A.5) and (A.7) we estimate

$$
\begin{aligned}
\|x\|_{1} & \leq\|x\|_{k-1}^{\frac{1}{k-1}}\|x\|_{0}^{\frac{k-2}{k-1}} \\
& \leq\|x\|_{k}^{\frac{k-2}{(k-1)^{2}}}\|x\|_{1}^{\frac{1}{(k-1)^{2}}}\|x\|_{0}^{\frac{k-2}{k-1}}
\end{aligned}
$$

Dividing both sides of this inequality by $\|x\|_{1}^{\frac{1}{(k-1)^{2}}}$ we obtain

$$
\|x\|_{1}^{\frac{k(k-2)}{(k-1)^{2}}} \leq\|x\|_{k}^{\frac{k-2}{(k-1)^{2}}}\|x\|_{0}^{\frac{k-2}{k-1}}
$$

or equivalently

$$
\|x\|_{1} \leq\|x\|_{k}^{\frac{1}{k}}\|x\|_{0}^{\frac{k-1}{k}} .
$$

This finishes the proof of $(A)_{k}$ in the case $j=1$. Now suppose that $j \geq 2$. In view of the induction hypothesis we have

$$
\|x\|_{j-1} \leq\|x\|_{k-1}^{\frac{j-1}{k-1}}\|x\|_{0}^{\frac{k-j}{k-1}}
$$

Shifting this inequality again we obtain

$$
\|x\|_{j} \leq\|x\|_{k}^{\frac{j-1}{k-1}}\|x\|_{1}^{\frac{k-j}{k-1}} .
$$

Combining this inequality with (A.8) we obtain

$$
\begin{aligned}
\|x\|_{j} & \leq\|x\|_{k}^{\frac{j-1}{k-1}}\|x\|_{1}^{\frac{k-j}{k-1}} \\
& \leq\|x\|_{k}^{\frac{j-1}{k-1}}\|x\|_{k}^{\frac{k-j}{(k-1) k}}\|x\|_{0}^{\frac{k-j}{k}} \\
& =\|x\|_{k}^{\frac{j}{k}}\|x\|_{0}^{\frac{k-j}{k}} .
\end{aligned}
$$

This finishes the proof of the induction step and hence of Step 2.

Step 3: We prove the theorem.

The theorem follows immediately from Step 2 by shifting the indices in the inequality.

The following example was used in the proof of the action-energy inequality.

Example A.2. For $i=1, j=4$, and $k=10$ we obtain

$$
\|x\|_{4} \leq C^{\prime}\|x\|_{10}^{\frac{4-1}{10-1}}\|x\|_{1}^{\frac{10-4}{10-1}}=C^{\prime}\|x\|_{10}^{\frac{1}{3}}\|x\|_{1}^{\frac{2}{3}} \quad \forall x \in E_{10}
$$

thus

$$
\|x\|_{4}^{3} \leq C\|x\|_{10}\|x\|_{1}^{2} \quad \forall x \in E_{10} .
$$

\section{Appendix B. Sobolev vs. interpolation}

Uniform exponential decay estimates for Morse theory can be found in Sch93, for Floer cylinders in Sal99, and for holomorphic strips in [RS01]. These proofs crucially use Sobolev inequalities. In this paper we do not use Sobolev inequalities but interpolation inequalities instead, see Appendix $\mathrm{A}$. They have the advantage that the dimension of the underlying spaces do not enter and therefore give rise to a unified approach to exponential decay estimates. 
In Fra09a it is proved that the sc-isomorphism type of the sc-space

$$
\ell^{f}=\left(\ell^{2} \supset \ell_{f}^{2} \subset \ell_{f^{2}}^{2} \supset \cdots\right)
$$

is uniquely described by the growth type of $f: \mathbb{N} \rightarrow \mathbb{R}_{+}$. That is,

$$
\ell^{f} \cong \ell^{g}
$$

if and only if there exists a constant $c>0$ such that

$$
\frac{1}{c} f \leq g \leq c f .
$$

Let $N$ be a closed manifold then Kang proved in [Kan11, Theorem A] that the sc-space

$$
L^{2}\left(N, \mathbb{R}^{d}\right) \supset W^{1,2}\left(N, \mathbb{R}^{d}\right) \supset W^{2,2}\left(N, \mathbb{R}^{d}\right) \supset \ldots
$$

is sc-isomorphic to $\ell^{f}$ with

$$
f(n)=n^{\frac{2}{\operatorname{dim} N}} .
$$

This reminds of the Sobolev inequalities which depend on the dimension of the source space. The advantage of the interpolation inequalities used in this article is that they are independent of the growth type of $f$. In particular, in the case of mapping spaces they are independent of the dimension of the source space. Thus, they lead to a unified approach to exponential decay estimates.

\section{REFERENCES}

[AM05] A. Abbondandolo and P. Majer, A Morse complex for infinite dimensional manifolds I, Adv. Math. 197 (2005), no. 2, 321-410.

[BL76] J. Bergh and J. Löfström, Interpolation spaces. An introduction, Springer-Verlag, Berlin, 1976, Grundlehren der Mathematischen Wissenschaften, No. 223.

[CZ83] C. C. Conley and E. Zehnder, The Birkhoff-Lewis fixed point theorem and a conjecture of V. I. Arnold, Invent. Math. 73 (1983), no. 1, 33-49.

[Flo88] A. Floer, The unregularized gradient flow of the symplectic action, Comm. Pure Appl. Math. 41 (1988), no. 6, 775-813.

[Fra09a] U. Frauenfelder, First steps in the geography of scale Hilbert structures, 2009, arXiv:0910.3980.

[Fra09b] - Fractal scale Hilbert spaces and scale Hessian operators, 2009, arXiv:0912.1154.

[GS05] A. R. Gaio and D. A. Salamon, Gromov-Witten invariants of symplectic quotients and adiabatic limits, J. Symplectic Geom. 3 (2005), no. 1, 55-159.

[HWZ07] H. Hofer, K. Wysocki, and E. Zehnder, A general Fredholm theory. I. : A splicing-based differential geometry, J. Eur. Math. Soc. (JEMS) 9 (2007), no. 4, 841-876.

[HWZ09a] — A general Fredholm theory. II. Implicit function theorems, Geom. Funct. Anal. 19 (2009), no. 1, 206-293.

[HWZ09b] _ A general Fredholm theory. III. Fredholm functors and polyfolds, Geom. Topol. 13 (2009), no. 4, 2279-2387.

[Kan11] J. Kang, Local invariant for scale structures on mapping spaces, 2011, arXiv:1108.0327.

[RS01] J. Robbin and D. A. Salamon, Asymptotic behaviour of holomorphic strips, Ann. Inst. H. Poincaré Anal. Non Linéaire 18 (2001), no. 5, 573-612.

[Sal99] D. A. Salamon, Lectures on Floer homology, Symplectic geometry and topology (Park City, UT, 1997), IAS/Park City Math. Ser., vol. 7, Amer. Math. Soc., Providence, RI, 1999, pp. 143-229.

[Sch93] M. Schwarz, Morse homology, Progress in Mathematics, vol. 111, Birkhäuser Verlag, Basel, 1993.

[Tri78] H. Triebel, Interpolation theory, function spaces, differential operators, North-Holland Mathematical Library, vol. 18, North-Holland Publishing Co., Amsterdam, 1978.

[Zil09] F. Ziltener, The invariant symplectic action and decay for vortices, J. Symplectic Geom. 7 (2009), no. $3,357-376$. 
Peter Albers, Mathematisches Institut, Westfälische Wilhelms-Universität Münster

E-mail address: peter.albers@wwu.de

Urs Frauenfelder, Department of Mathematics and Research Institute of Mathematics, Seoul National University

E-mail address: frauenf@snu.ac.kr 